AperTO - Archivio Istituzionale Open Access dell'Università di Torino

\title{
Relaxed schemes for nonlinear evolutionary PDEs
}

\section{This is the author's manuscript}

Original Citation:

Availability:

This version is available http://hdl.handle.net/2318/93510

since 2017-03-13T12:00:08Z

Publisher:

AMS

Terms of use:

Open Access

Anyone can freely access the full text of works made available as "Open Access". Works made available under a Creative Commons license can be used according to the terms and conditions of said license. Use of all other works requires consent of the right holder (author or publisher) if not exempted from copyright protection by the applicable law. 


\title{
Relaxed schemes for nonlinear evolutionary PDEs
}

\author{
F. Cavalli, G. Naldi, G. Puppo, and M. Semplice
}

\begin{abstract}
In this work we present a class of relaxed schemes for non linear convection diffusion problems, which can tackle also the cases of degenerate diffusion and of convection dominated regimes. These schemes can achieve any order of accuracy and they give non-oscillatory solutions even in the presence of singularities.

Relaxation approximations to non-linear PDE's are based on the replacement of the original PDE with a semi-linear hyperbolic system of equations, with a stiff source term, tuned by a relaxation parameter $\varepsilon$. When $\varepsilon \rightarrow 0$ the system relaxes onto the original PDE. A consistent discretization of the relaxation system for $\varepsilon=0$ yields a consistent discretization of the original PDE. The advantage of this procedure is that the numerical scheme obtained in this fashion does not need approximate Riemann solvers for the convective term, still enjoying the robustness of upwind discretizations. We also present a numerical test for a strongly degenerate convection diffusion equation.
\end{abstract}

\section{Introduction}

Relaxation approximations have been successfully employed as a basis to construct novel numerical schemes for different kinds of partial differential equations (PDEs). The origin of these schemes lies in the relaxation of kinetic equations towards fluid dynamic limits. Jin-Xin relaxation system [JX95] in its characteristic form can be viewed as a simplified kinetic system relaxing onto a conservation equation (see also [JL96, CJR97]). Exploiting the diffusive limit of kinetic systems onto parabolic equations, relaxation schemes for convection-diffusion equations can be built [NPT02, JPT98]. Relaxation systems can be obtained from different collisional kernels: [ADN00, LN02] use BGK kernels and [Suc01, BKPS08] exploit the lattice Boltzmann approach.

We start from a fairly general relaxation system and give consistency conditions ensuring that the limit equation is the desired partial differential equation (PDE) [CNPS]. This class includes the schemes of [ADN00, LN02], but also some new relaxation schemes which are discussed in this work (see also [CNPS09]).

1991 Mathematics Subject Classification. Primary 65M25,65M06.

Key words and phrases. relaxation schemes, convection-diffusion, degenerate parabolic equations.

This work was partially supported by PRIN2007 grant "Modellistica numerica per il calcolo scientifico ed applicazioni avanzate". 
The main idea at the base of relaxation approximations is to replace the original nonlinear PDE with a semilinear hyperbolic system with a stiff source term and a relaxation parameter $\varepsilon$. Since the resulting hyperbolic differential operator is linear with constant coefficients, the mapping to characteristic variables is constant in time and therefore convection can be computed with upwinding, without using Riemann solvers. The stiffness in the source term (in the limit $\varepsilon \rightarrow 0$ ) is treated with a suitable time integrator, as Implicit-Explicit Runge-Kutta schemes.

In this paper we consider relaxation systems in the following general form

$$
s_{t}+A s_{x}+\frac{1}{\varepsilon} B s_{x}=\frac{1}{\varepsilon}(h(u)-C s),
$$

with $s \in \mathbf{R}^{d}$ and suitable constant matrices $A, B, C \in \mathbf{R}^{d \times d}$. This framework encompasses, among others, the Jin-Xin relaxation approximation of conservation laws [JX95], the diffusive relaxation schemes of [JPT98] and the BGK kinetic approximations of [ADN00, LN02].

As an example, consider the one-dimensional Maxwell-Cattaneo system derived from the kinetic Goldstein-Taylor model of [Tay21, Gol51]

$$
\left\{\begin{array}{l}
u_{t}+v_{x}=0 \\
v_{t}+\frac{1}{\varepsilon} u_{x}=-\frac{1}{\varepsilon} v
\end{array}\right.
$$

that corresponds to the choices

$$
s=\left[\begin{array}{l}
u \\
v
\end{array}\right] \quad A=\left[\begin{array}{ll}
0 & 1 \\
0 & 0
\end{array}\right] \quad B=\left[\begin{array}{ll}
0 & 0 \\
1 & 0
\end{array}\right] \quad C=\left[\begin{array}{ll}
1 & 0 \\
0 & 1
\end{array}\right] \quad h(u)=\left[\begin{array}{l}
u \\
0
\end{array}\right]
$$

In the limit $\varepsilon \rightarrow 0$, the second equation of (1.2) relaxes onto $u_{x}=-v$, while the first one relaxes onto $u_{t}+v_{x}=0$ and thus $u$ formally converges to the solution of the linear diffusion equation $u_{t}=u_{x x}$.

In [CNPS07] we studied the system corresponding to the choices

$s=\left[\begin{array}{c}u \\ v \\ w\end{array}\right] \quad A=\left[\begin{array}{lll}0 & 1 & 0 \\ 0 & 0 & 0 \\ 0 & 1 & 0\end{array}\right] \quad B=\left[\begin{array}{lll}0 & 0 & 0 \\ 0 & 0 & 1 \\ 0 & 0 & 0\end{array}\right] \quad C=\left[\begin{array}{lll}1 & 0 & 0 \\ 0 & 1 & 0 \\ 0 & 0 & 1\end{array}\right] \quad h(u)=\left[\begin{array}{c}u \\ 0 \\ p(u)\end{array}\right]$

which corresponds to the relaxation system

$$
\left\{\begin{array}{l}
\partial_{t} u+\partial_{x} v=0 \\
\partial_{t} v+\frac{1}{\varepsilon} \partial_{x} w=\frac{1}{\varepsilon}(-v) \\
\partial_{t} w+\partial_{x} v=\frac{1}{\varepsilon}(p(u)-w)
\end{array}\right.
$$

When $\varepsilon \rightarrow 0$, the first equation relaxes onto $\partial_{t} u=\partial_{x x} p(u)$, which includes for example the porous media equation when $p(u)=u^{m}$ with $m>1$.

Finally, to obtain a relaxation approximation for the convection-diffusion equation $\partial_{t} u+\partial_{x} f(u)=\partial_{x x} p(u)$ we can choose

$$
s=\left[\begin{array}{c}
u \\
v \\
w
\end{array}\right] \quad A=\left[\begin{array}{ccc}
0 & 1 & 0 \\
a^{2} & 0 & 0 \\
0 & b^{2} & 0
\end{array}\right] \quad B=\left[\begin{array}{lll}
0 & 0 & 0 \\
0 & 0 & 1 \\
0 & 0 & 0
\end{array}\right] \quad C=\left[\begin{array}{lll}
1 & 0 & 0 \\
0 & 1 & 0 \\
0 & 0 & 1
\end{array}\right] \quad h(u)=\left[\begin{array}{c}
u \\
f(u) \\
p(u)
\end{array}\right]
$$

obtaining the relaxation system

$$
\left\{\begin{array}{l}
\partial_{t} u+\partial_{x} v=0 \\
\partial_{t} v+a^{2} \partial_{x} u+\frac{1}{\varepsilon} \partial_{x} w=\frac{1}{\varepsilon}(f(u)-v) \\
\partial_{t} w+b^{2} \partial_{x} v=\frac{1}{\varepsilon}(p(u)-w)
\end{array}\right.
$$


More applications and generalizations to the multidimensional diffusion and convectiondiffusion equations are described in [CNPS07, CNPS09, CNPS].

In the following we describe the numerical discretization of the relaxation system. First we describe the semidiscretization in time and then the space discretization (section 2). Then we investigate the stability of the first order numerical schemes derived from the relaxation system (1.4) (section 3) and we test a few of the proposed schemes on a strongly degenerate diffusion equation (section 4 ).

\section{Numerical schemes}

The form of system (1.1) is not suitable for numerical integration, because of the stiffness contained in the linear convective term, which would result in a prohibitively restrictive CFL condition. As in [NPT02] and [CNPS07], we remove the stiffness from the convective term adding and subtracting the quantity $\phi^{2} B s_{x}$, thus rewriting (1.1) in the form:

$$
s_{t}+\left(A+\phi^{2} B\right) s_{x}=\frac{1}{\varepsilon}\left(h(u)+\left(\varepsilon \phi^{2}-1\right) B s_{x}-C s\right) .
$$

We point out that the choice of a relaxation system for a given PDE is by no means unique. On the other hand, different relaxation systems give rise to different numerical schemes: thus it is important to characterize the systems which result in the most efficient schemes. In [CNPS] we showed that the following consistency conditions ensure that (2.1) relaxes onto the convection diffusion equation $\partial_{t} u+$ $\partial_{x} f(u)=\partial_{x x} p(u):$

$$
\begin{aligned}
& A+\phi^{2} B \quad \text { is diagonalizable with real eigenvalues } \forall \phi \\
& s_{1}=u \quad\left[C^{-1} h(u)\right]_{1}=u \\
& {[B]_{1}=\overrightarrow{0}} \\
& {\left[A C^{-1} h\right]_{1}=f(u)} \\
& {\left[A C^{-1} B C^{-1} h\right]_{1}=p(u)} \\
& \left(C^{-1} B\right)^{l}=0, l \geq 2 .
\end{aligned}
$$

Here we denoted with $[W]_{1}$ the first row of the matrix (or vector) $W$. Within this framework several schemes can be obtained.

The system (2.1) can be integrated with any order of accuracy. The technical details and the description of the high order schemes were studied in [CNPS] and will be just sketched here.

2.1. Time semidiscretization. Let us rewrite system (2.1) as

$$
s_{t}+A_{\phi} s_{x}=\frac{1}{\varepsilon} H_{\phi}(s)
$$

where $A_{\phi}=A+\phi^{2} B$ and $H_{\phi}(s)=h(u)+\left(\varepsilon \phi^{2}-1\right) B s_{x}-C s$.

For the semi-discretization of (2.3) in time, the choice of an IMEX scheme (Implicit-Explicit Runge-Kutta pair) [ARS97, KC03, PR05] is natural, given the stiffness of the right hand side. Let us consider for simplicity a uniform time step $\Delta t$ and denote with $s^{n}$ the numerical approximation of the variable $s$ at time $t_{n}=n \Delta t$, for $n=0,1, \ldots$ We employ a $\nu$-stages IMEX scheme and include the $1 / \varepsilon$ 
terms in the implicit part, obtaining

$$
\begin{aligned}
& s^{n+1}=s^{n}-\Delta t \sum_{i=1}^{\nu} \tilde{b}_{i} \partial_{x}\left(A_{\phi} s^{(i)}\right)+\frac{\Delta t}{\varepsilon} \sum_{i=1}^{\nu} b_{i} H_{\phi}\left(s^{(i)}\right) \\
& s^{(i)}=s^{n}-\Delta t \sum_{k=1}^{i-1} \tilde{a}_{i k} \partial_{x}\left(A_{\phi} s^{(k)}\right)+\frac{\Delta t}{\varepsilon} \sum_{k=1}^{i} a_{i k} H_{\phi}\left(s^{(k)}\right)
\end{aligned}
$$

Here $\left(a_{i k}, b_{i}\right)$ and $\left(\tilde{a}_{i k}, \tilde{b}_{i}\right)$ are the two Butcher's tableaux of, respectively, the diagonally implicit and the explicit parts of the IMEX pair. Note that $(2.4 \mathrm{~b})$ defines implicitly the $i^{\text {th }}$ stage $s^{(i)}$ and (2.4a) computes $s$ at time $t^{n+1}$.

In this work we use the so-called relaxed schemes, that are obtained by letting $\varepsilon \rightarrow 0$ in the equations (2.4). In the limit we have that

$$
\forall i: \quad \sum_{k=1}^{i} a_{i k}\left[H\left(s^{(k)}\right)\right]_{j}=0
$$

Then we obtain $\left[H\left(s^{(k)}\right)\right]_{j}=0$ for each component $j$, which simplifies $(2.4)$ to

$$
s^{n+1}=s^{n}-\Delta t \sum_{i=1}^{\nu} \tilde{b}_{i} \partial_{x}\left(A_{\phi} s^{(i)}\right) \quad s^{(i)}=s^{n}-\Delta t \sum_{k=1}^{i-1} \tilde{a}_{i k} \partial_{x}\left(A_{\phi} s^{(k)}\right)
$$

We remark that we need to advance in time only the first component $\left[s^{(i)}\right]_{1}=u$, which is the only physical variable we need to update. Thus the relaxed scheme becomes an alternation of relaxation steps

$$
H\left(s^{(i)}\right)=0
$$

and transport steps

$$
s_{1}^{(i)}=s_{1}^{n}-\Delta t \sum_{k=1}^{i-1} \tilde{a}_{i k} \partial x\left(A_{\phi} s^{(i)}\right)
$$

followed by the computation of $s_{1}^{n+1}=u^{n+1}$ according to (2.4a):

$$
u^{n+1}=u^{n}-\Delta t \sum_{i=1}^{\nu} \tilde{b}_{i} \partial_{x}\left(A_{\phi} s^{(i)}\right)_{1}
$$

We point out that in the examples considered in section 1 , the relaxation step $H\left(s^{(i)}\right)=0$ can be solved by back substitution, determining values $\left[s^{(i)}\right]_{j}$ for $j \geq 2$ without the need to solve a nonlinear system. Moreover, it is important to realize that there is no need to compute the components $s_{k}^{(i)}$ for $k \geq 2$ in the transport steps, since they would be overridden by the implicit computation (2.6a) for the next stage.

We point out that only the explicit part of the previous Runge-Kutta pair is involved in updating the solution $u$. In fact, given a relaxation system and an explicit RK scheme $\left(\tilde{a}_{i k}, \tilde{b}_{i}\right)$, it is possible to construct an associated relaxed scheme, provided that there exists a diagonally implicit RK scheme that forms an IMEX pair of the same order. 
2.2. Space discretization. The main task of the space discretization is the evaluation of the boundary extrapolated data along characteristic variables. Here we describe the one-dimensional case, the generalization to multi-dimension being straightforward by dimensional splitting. Let $s_{j}^{n}$ be the value of the vector function $s(x, t)$ at time $t^{n}$ at $x_{j}$, the centre of the $j^{\text {th }}$ computational cell. We consider a fully discrete scheme in conservation form

$$
s_{j}^{n+1}=s_{j}^{n}-\Delta t \sum_{i=1}^{\nu} \tilde{b}_{i}\left(F_{j+1 / 2}^{(i)}-F_{j-1 / 2}^{(i)}\right)
$$

where $F_{j+1 / 2}^{(i)}$ are the numerical fluxes. In order to minimize dissipation we use Godunov fluxes, which in this case coincide with upwinding. Let $R$ be an invertible constant matrix that diagonalizes $A_{\phi}$, i.e. $R^{-1} A_{\phi} R=\Lambda$. The $i^{\text {th }}$ component of $S=R^{-1} s$ satisfies an advection equation with constant speed $\Lambda_{i i}$ and the numerical flux is

$$
F_{j+1 / 2}^{(i)}=R \Lambda S_{j+1 / 2}^{(i), \sigma\left(\lambda_{i}\right)}
$$

where $\sigma\left(\lambda_{i}\right)=-\operatorname{sign}\left(\lambda_{i}\right)$ and $S_{j+1 / 2}^{(i), \pm}$ are the boundary extrapolated data at the edges of the $j^{\text {th }}$ cell, computed from the point values $S^{(i)}=R^{-1} s^{(i)}$ using a nonoscillatory procedure of suitable order.

The accuracy of the scheme depends on the accuracy of the reconstruction of the boundary extrapolated data. In order to get a first order scheme one may use a piecewise constant reconstruction such that $S_{j+1 / 2}^{-}=S_{j}$ and $S_{j+1 / 2}^{+}=S_{j+1}$. ENO or WENO reconstructions of appropriate accuracy are a valid choice for higher order schemes [SO89].

Finally we wish to emphasize that, although the scheme is based on a system of three or more equations, the construction is used only to select the correct upwinding for the fluxes of the relaxed scheme and the computational cost of each time step is not affected by the number of equations in the relaxation system. The computational cost of the numerical approximation depends on the properties of the $A_{\phi}$ and $R$ matrices. Since only the first component of (2.8) has to be evaluated, the space reconstructions and corresponding fluxes computation have to be performed only for the components $S_{k}$ such that $R_{1 k} \neq 0$ and $\Lambda_{k k} \neq 0$. Hence relaxation schemes leading to $A_{\phi}$ matrices with zero eigenvalues and $R$ matrices with the most zero elements in the first row are to be preferred (see [CNPS] for two examples of relaxation systems with four equations that give rise to two relaxed schemes whose computational cost differ by a factor 2).

For convection-diffusion, the relaxation step requires the computation of gradients of some components of $s^{(i)}$. These are computed by centered finite-difference formulas of suitable order, except at cells close to the boundary, where we employ asymmetric formulas of the same order observing no loss of accuracy.

Finally, boundary conditions are enforced by extrapolating the approximate solution and/or the stage values $u_{j}^{(i)}$ to one ghost point located outside the domain before the computation of each Runge-Kutta stage.

\section{Three velocities models}

As a prototypical example, we consider the first order numerical relaxed schemes obtained from the relaxation system (1.4). Using the IMEX pair that combines the 
explicit and the implicit Euler scheme, one gets

$$
\begin{aligned}
u_{j}^{n+1}=u_{j}^{n}- & \frac{\Delta t}{2 h}\left(v_{j+1}^{n}-v_{j-1}^{n}\right) \\
& +\frac{a^{2} \Delta t}{2 h \mu}\left(u_{j-1}^{n}-2 u_{j}^{n}+u_{j+1}^{n}\right)-\frac{\phi^{2} \Delta t}{2 h \mu}\left(w_{j-1}^{n}-2 w_{j}^{n}+w_{j+1}^{n}\right)
\end{aligned}
$$

where $\mu=\sqrt{a^{2}+b^{2} \phi^{2}}$. Substituting $v_{j}^{n}=f\left(u_{j}^{n}\right)-\left(w_{j+1}^{n}-w_{j-1}^{n}\right) / 2 h$, we get

$$
\begin{aligned}
& u_{j}^{n+1}=u_{j}^{n}-\frac{\Delta t}{2 h}(\left.f\left(u_{j+1}^{n}\right)-f\left(u_{j-1}^{n}\right)\right)+\frac{a^{2} \Delta t}{2 h}\left(u_{j-1}^{n}-2 u_{j}^{n}+u_{j+1}^{n}\right) \\
&+\frac{\Delta t}{2 h}\left[\frac{w_{j-2}^{n}-2 w_{j}^{n}+w_{j+2}^{n}}{2 h}+\frac{\phi^{2}}{\mu}\left(w_{j-1}^{n}-2 w_{j}^{n}+w_{j+1}^{n}\right)\right]
\end{aligned}
$$

We now consider the stability of the above scheme. In particular we focus on the monotonicity of the scheme, i.e. we require that the derivatives of $u_{j}^{n+1}$ with respect to $u_{j+k}^{n}$ be positive for $k=-2, \ldots, 2$. We note that monotone schemes are total variation diminishing and thus convergent.

One easily checks that the scheme obtained from (3.2) is monotone provided that the following conditions hold in each computational cell:

$$
\begin{cases}\frac{a^{2}+\phi^{2} p^{\prime}}{\sqrt{a^{2}+\phi^{2} b^{2}}}>\left|f^{\prime}\right| & \text { (subcharacteristic) } \\ \Delta t \leq \frac{h}{\frac{a^{2}+p^{\prime} \phi^{2}}{\sqrt{a^{2}+\phi^{2} b^{2}}}+\frac{p^{\prime}}{2 h}} & \text { (time step) }\end{cases}
$$

The first inequality is a kind of subcharacteristic condition, while the second one contains the stability restriction of the time step $\Delta t$. We observe that, using the first relation, one may write the time step restriction as

$$
\Delta t \leq \frac{h}{V+\frac{D}{2 h}}
$$

where $V$ and $D$ are upper bounds for $\left|f^{\prime}\right|$ and $p^{\prime}$ respectively. In particular the scheme is stable under the hyperbolic condition $\Delta t \sim h / V$ in the case of convectiondominated problems, and under the parabolic constraint $\Delta t \sim h^{2} / D$ for diffusion dominated cases.

For higher order schemes we obtain similar constraints through Von Neumann analysis applied to the linearization of the scheme (see [CNPS07]).

\section{Numerical test}

We consider the following evolutionary problem

$$
\partial_{t} u+(W-V) \partial_{x} u=\partial_{x x} \beta(u)
$$

where $\beta(u)=(u-1)_{+}-u_{-}$with initial data

$$
u_{0}(x)= \begin{cases}\gamma_{2} \frac{e^{V x}-1}{V}+1 & x>0 \\ \gamma_{1} \frac{e^{V x}-1}{V} & x<0\end{cases}
$$

where $\gamma_{i}$ are constants and $V=\gamma_{2}-\gamma_{1}$. The problem has the exact solution $u(t, x)=u_{0}(x+W t)$ which represents a discontinuous travelling wave with velocity $W$. In the case $W=V$ this is a classical Stefan problem (see e.g. [NPV91]). Even when $W \neq V$, this is a strongly degenerate diffusion problem, since $\beta(u)$ vanishes over the interval $[0,1]$. 

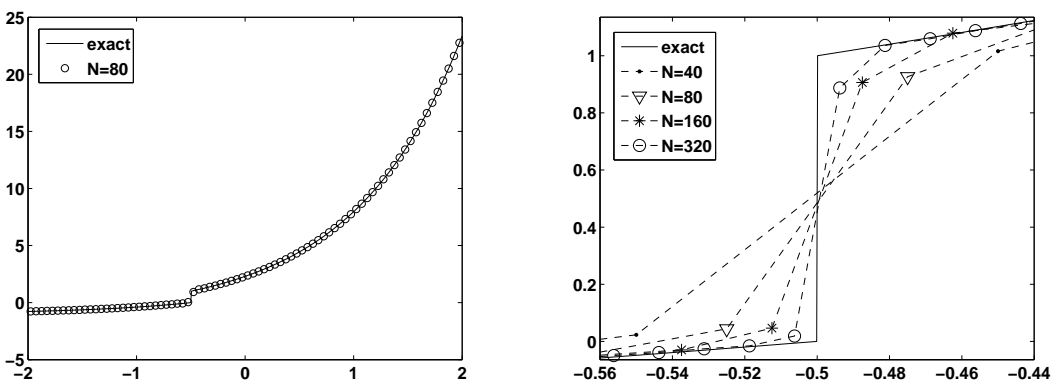

Figure 1. Stefan problem (4.1) with $W=V$. On the left the exact solution and the numerical solution with 80 points at $t=0.5$. On the right, comparison of the numerical solutions around the discontinuity with different grids.
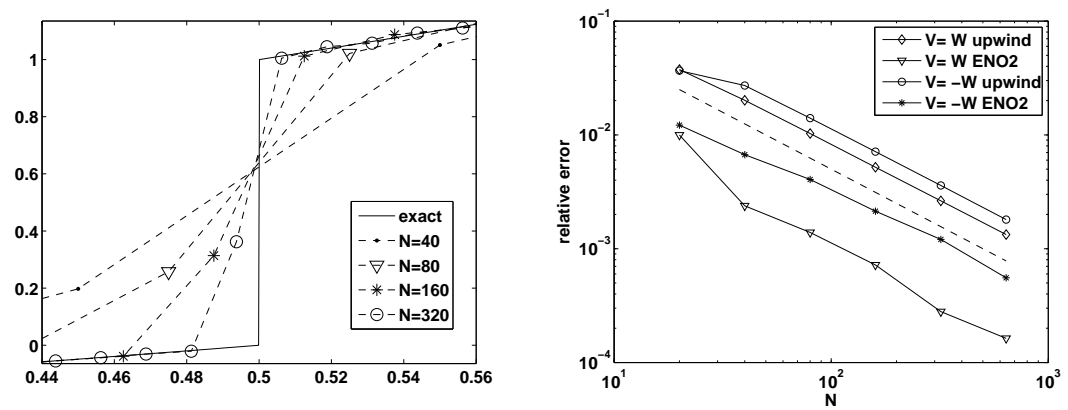

Figure 2. Stefan problem (4.1). On the left comparison of the numerical solutions around the discontinuity with different grids, in the case $W=-V$. On the right, relative $L_{1}$ error for the numerical integration of the Stefan problem with $W=V$ and $W=-V$.

We integrated numerically the above problem on the domain $[-2,2]$ up to time $t=0.5$, setting $a=b=\phi=1$ in the definition of the numerical scheme obtained from (2.6) with the first order scheme (3.2) and with the second order ENO space reconstruction and Euler time integration.

In Figure 1 we show the exact and the second order numerical solution in the left panel, for the classical Stefan case $\left(\gamma_{2}=2, \gamma_{1}=1, W=V\right)$. On the right there is an enlargement of the area around $x=-0.5$ (the final position of the step discontinuity) showing the numerical solutions obtained with different number of computational cells (from 40 to 320 ).

In Figure 2 we consider instead the case with the convective term: we take $W=-V$. The left panel is an enlargement of the area around the final position of the discontinuity $(x=0.5)$. The right panel shows the convergence rate of the relative $L_{1}$ errors for both tests (see also Table 1 for the second order scheme). The dashed line is the reference for first order convergence, which is the expected result 


\begin{tabular}{|c|l|l|l|l|}
\hline $\mathrm{N}$ & \multicolumn{2}{|c|}{$W=V$} & \multicolumn{2}{c|}{$W=-V$} \\
\hline 20 & $9.9759 \mathrm{e}-03$ & rate & $1.2191 \mathrm{e}-02$ & rate \\
\hline 40 & $2.3825 \mathrm{e}-03$ & 2.07 & $6.7032 \mathrm{e}-03$ & 0.86 \\
\hline 80 & $1.3886 \mathrm{e}-03$ & 0.78 & $4.0535 \mathrm{e}-03$ & 0.73 \\
\hline 160 & $7.1996 \mathrm{e}-04$ & 0.95 & $2.1373 \mathrm{e}-03$ & 0.92 \\
\hline 320 & $2.7977 \mathrm{e}-04$ & 1.36 & $1.2055 \mathrm{e}-03$ & 0.83 \\
\hline 640 & $1.6309 \mathrm{e}-04$ & 0.78 & $5.5525 \mathrm{e}-04$ & 1.12 \\
\hline \multicolumn{2}{|c|}{ average rate } & 1.14 & & 0.88 \\
\hline
\end{tabular}

TABLE 1. Stefan problem (4.1). Relative error $\left\|u-u_{h}\right\|_{1} /\|u\|_{1}$ for the ENO2-based scheme.

since the exact solution is discontinuous. Nonetheless the graph shows the increase in accuracy obtained using a higher order scheme.

\section{Conclusions}

We presented a general form for relaxation systems for nonlinear convection diffusion equations, giving conditions that ensures consistency with the original PDE. Deriving relaxed schemes in such a general setting, enlightens the features of the method and the properties of the relaxation system that influence the computational cost of the schemes.

Even if in this paper we dealt only with a one-dimensional convection-diffusion equation, we wish to point out that this method is not restricted to one-dimensional evolutionary equations nor to second order equations. Equations set in more than one spatial dimension can in fact be treated by dimensional splitting ([CNPS07, CNPS]) and in principle also differential operators of order higher than two can be treated by relaxation techniques.

In future works we plan to analyze further the nonlinear stability of high order schemes and to study the extension of this method to reaction diffusion equations and to nonlinear fourth order diffusion equations, like the thin film equation.

\section{References}

[ADN00] D. Aregba-Driollet and R. Natalini, Discrete kinetic schemes for multidimensional systems of conservation laws, SIAM J. Numer. Anal. 37 (2000), no. 6, 1973-2004.

[ARS97] U. Asher, S. Ruuth, and R.J. Spiteri, Implicit-explicit Runge-Kutta methods for time dependent Partial Differential Equations, Appl. Numer. Math. 25 (1997), 151-167.

[BKPS08] Mapundi Banda, Axel Klar, Lorenzo Pareschi, and Mohammed Seaïd, LatticeBoltzmann type relaxation systems and high order relaxation schemes for the incompressible Navier-Stokes equations, Math. Comp. 77 (2008), no. 262, 943-965.

[CJR97] Russel E. Caflisch, Shi Jin, and Giovanni Russo, Uniformly accurate schemes for hyperbolic systems with relaxation, SIAM J. Numer. Anal. 34 (1997), no. 1, 246-281.

[CNPS] F. Cavalli, G. Naldi, G. Puppo, and M. Semplice, Relaxation schemes for nonlinear convection-diffusion problems., Numerical methods for balance laws (G. Puppo and G. Russo, eds.), Submitted.

[CNPS07] _ High-order relaxation schemes for non linear degenerate diffusion problems, SIAM Journal on Numerical Analysis 45 (2007), no. 5, 2098-2119.

[CNPS09] _ A family of relaxation schemes for nonlinear convection diffusion problems, Commun. Comput. Phys. 5 (2009), no. 2-4, 532-545.

[Gol51] S. Goldstein, On diffusion by discontinuous movements, and on the telegraph equation, Quart. J. Mech. Appl. Math. 4 (1951), 129-156. 
[JL96] S. Jin and C. D. Levermore, Numerical schemes for hyperbolic conservation laws with stiff relaxation terms, J. Comput. Phys. 126 (1996), no. 2, 449-467.

[JPT98] S. Jin, L. Pareschi, and G. Toscani, Diffusive relaxation schemes for multiscale discretevelocity kinetic equations, SIAM J. Numer. Anal. 35 (1998), no. 6, 2405-2439.

[JX95] S. Jin and Z. Xin, The relaxation schemes for systems of conservation laws in arbitrary space dimensions, Comm. Pure and Appl. Math. 48 (1995), 235-276.

[KC03] C. A. Kennedy and M. H. Carpenter, Additive Runge-Kutta schemes for convectiondiffusion-reaction equations, Appl. Numer. Math. 44 (2003), no. 1-2, 139-181.

[LN02] C. Lattanzio and R. Natalini, Convergence of diffusive BGK approximations for nonlinear strongly parabolic systems, Proc. Roy. Soc. Edinburgh Sect. A 132 (2002), no. 2, 341-358.

[NPT02] G. Naldi, L. Pareschi, and G. Toscani, Relaxation schemes for partial differential equations and applications to degenerate diffusion problems, Surveys Math. Indust. 10 (2002), no. 4, 315-343.

[NPV91] R. H. Nochetto, M. Paolini, and C. Verdi, An adaptive finite element method for twophase Stefan problems in two space dimensions. I. Stability and error estimates, Math. Comp. 57 (1991), no. 195, 73-108, S1-S11.

[PR05] L. Pareschi and G. Russo, Implicit-explicit Runge-Kutta schemes and applications to hyperbolic systems with relaxation, J. Sci. Comp. 25 (2005), 129-155.

[SO89] C. Shu and S. Osher, Efficient implementation of essentially nonoscillatory shockcapturing schemes. II, J. Comput. Phys. 83 (1989), no. 1, 32-78.

[Suc01] S. Succi, The lattice Boltzmann equation for fluid dynamics and beyond, Numerical Mathematics and Scientific Computation, The Clarendon Press Oxford University Press, New York, 2001, Oxford Science Publications.

[Tay21] G. I. Taylor, Diffusion by continuous movements, Proc. London Math. Soc. 20 (1921), 196-212.

Dipartimento di Matematica, Università degli Studi di Milano, Via Saldini 50, 20133 Milano (Italy)

Current address: Dipartimento di Ingegneria, Università di Brescia.

E-mail address: fausto.cavalli@ing.unibs.it

Dipartimento di Matematica, Università degli Studi di Milano, Via Saldini 50, 20133 Milano (ItALY)

E-mail address: naldi@mat.unimi.it

Dipartimento di Matematica, Politecnico di Torino, corso Duca degli Abruzzi, 24 , 10129 TORINO, ITALY

E-mail address: gabriella.puppo@polito.it

Dipartimento di Matematica, Università degli Studi di Milano, Via Saldini 50, 20133 Milano (Italy)

E-mail address: semplice@mat.unimi.it 